\title{
L'intérêt pour les petits producteurs fonde-t-il la consommation de produits équitables ?
}

\author{
Maud Daniel ${ }^{1}$ \\ Nicolas Bricas ${ }^{2}$ \\ Lucie Sirieix ${ }^{3}$
}

${ }^{1}$ Cirad/ Supagro Montpellier 45 bis, avenue de la Belle Gabrielle 94736 Nogent sur Marne

France

<maud.daniel@gmail.com>

${ }^{2}$ Cirad UMR Moisa

Montpellier

France

<nicolas.bricas@cirad.fr>

${ }^{3}$ Montpellier Supagro

Montpellier

France

<sirieix@supagro.inra.fr>

\begin{abstract}
Résumé
Cette étude s'intéresse aux liens entre les motivations des consommateurs à acheter des produits issus du commerce équitable et les deux mentions "Commerce équitable " et "Petits producteurs" souvent juxtaposées sur les emballages. Deux enquêtes, qualitative puis quantitative, sur 228 individus, nous permettent de montrer que la perception que les individus ont de ces produits est moins en cohérence avec les principes altruistes du commerce équitable qu'avec la mention "petits producteurs ", qui a une connotation de soutien aux plus pauvres, mais a aussi des motivations moins altruistes, comme la recherche d'un produit au meilleur goût ou plus authentique.
\end{abstract}

Mots clés : commerce équitable ; comportement du consommateur ; enquête auprès des consommateurs ; label de qualité ; producteur.

Thèmes : alimentation, consommation, nutrition ; économie et développement rural ; transformation, commercialisation.

\section{Abstract \\ Do consumers buy Fair Trade products because they come from "small producers"?}

In this study, we explore the links between consumer motives to purchase fair trade products and the two "fair trade" and "small producers" mentions often juxtaposed on the products. Two qualitative and quantitative surveys show that the perceptions of some consumers about these products are less linked to moral and fundamental principles of fair trade than to the mention of "small producers" which connotes the supporting of poor producers as well as a product made with care which is tastier and more authentic.

Key words: consumer behaviour; consumer surveys; fair trade; producers; quality labels.

Subjects: economy and rural development; food, consumption, nutrition; processing, marketing. epuis quelques années, l'offre et la demande des produits issus de l'agriculture biologique $(\mathrm{AB})$ ou du commerce équitable (CE) se sont développées et ces types de produits sont apparus dans la grande distribution. Les médias se font désormais largement l'écho des initiatives du secteur agroalimentaire dans ce domaine. Ce phénomène est d'ailleurs présenté par les médias comme un signe des préoccupations éthiques croissantes de la société. Pour de nombreux auteurs, la croissance du marché de ces produits manifeste aussi une contestation du système agroalimentaire "industrialisé " ou "productiviste ", une critique "artiste" du capitalisme (Boltanski et Chiapello, 1999) et l'intérêt porté à l'émergence d'alternatives à ce système (Seyfang, 2006 ; Micheletti et al., 2004 ; Stolle et al., 2005).

\section{Acheter des produits " équitables " : l'expression systématique d'une contestation ou d'un engagement ?}

Dans le cas des produits issus de l'agriculture biologique, Henson et Traill (2000) ont montré, aux États-Unis et au 
Royaume-Uni, un décalage entre les motivations altruistes et éthiques des promoteurs initiaux de cette agriculture et l'intérêt plus égoïste des consommateurs. Les produits biologiques sont moins achetés par les consommateurs pour des raisons environnementales que pour la santé ou pour le plaisir gustatif qu'ils procurent. Les produits issus du commerce équitable connaissent-ils le même décalage?

C'est ce qu'explorent Tagbata et Sirieix (2010) à propos du chocolat équitable. Ils montrent que, même si les préoccupations éthiques des individus sont importantes, la qualité gustative reste un critère de choix dominant. De Ferran et Grunert (2007) vont plus loin et montrent que les produits équitables sont achetés pour atteindre trois objectifs différents, qui peuvent être altruistes, mais aussi plus égoïstes : un désir d'équité dans les relations humaines, le souhait de protéger l'environnement et sa propre santé, et enfin la recherche de produits de qualité, d'hédonisme et de plaisir.

\section{Quels sont, pour le consommateur, les liens entre les mentions " Petits producteurs " et « Commerce équitable "?}

Avec le développement rapide de la demande en produits équitables, certains opérateurs s'interrogent sur la possibilité de labelliser "Équitable " de grandes plantations patronales qui s'engageraient à une meilleure rémunération de leur main-d'œeuvre salariée. Une telle démarche leur semble cependant risquée et ces opérateurs s'interrogent sur le lien que font les consommateurs entre la mention commerce équitable et le type d'entreprise de production concerné.

Dans ce contexte, nous proposons d'étudier ici la relation entre deux attributs souvent mis en avant sur les mêmes produits, les mentions "Commerce équitable" (CE) et "Petits producteurs " (PP). En effet, un relevé des mentions figurant sur les paquets de produits équitables dans 10 circuits de distribution différents nous a permis de mettre en évidence la fréquence de cette dernière mention. Sur 16 marques de café équitable observées, huit emballages font nettement référence à une production limitée en volume ou mentionnent une origine de "petits producteurs ", " petits exploitants " ou " petits cultivateurs ". Par ailleurs, 10 utilisent sur leurs emballages une photo ou un dessin de producteurs dans une version idéalisée (tenue traditionnelle, sourire, chapeau de paille...). Si la plupart des marques participent par leur stratégie à véhiculer les principes du commerce équitable, la communication se fait donc aussi sur l'image des petits producteurs. Comme l'a montré Ruwet (2007), les consommateurs engagés opposent souvent "la petite production fermière" à "l'industrie agricole". Alors que, dans leurs discours, la première est associée à l'humanisation des filières et à la consommation durable, la seconde est associée à un système uniformisé de grande ampleur, au profit et à la consommation de masse.

\section{La mention PP : un attribut d'authenticité}

Si l'éthique est devenue un outil stratégique majeur pour les entreprises, l'authenticité peut aussi être mobilisée, comme l'ont relevé Warnier (1994) ou Camus (2007). Ce dernier montre que l'authenticité perçue d'un objet peut se définir en référence à une époque, à l'autorité de son auteur, au lieu d'origine de la fabrication, à une origine culturelle ou traditionnelle, à la nature, ou à une technique ou technologie particulière. Ainsi, les mentions "Petits producteurs ", "Respect de l'environnement ", les cartes illustrant l'origine du produit et les histoires racontées au dos des emballages sont autant d'attributs qui construisent un contexte dit " expérientiel " de la consommation de ces produits, c'est-à-dire un contexte "dans lequel le consommateur s'immerge pour avoir accès à une expérience" (Carù et Cova, 2006), ici perçue comme authentique.

La mention " petits producteurs " présente sur de nombreux produits équitables peut donc répondre à deux préoccupations actuelles : la quête d'authenticité des produits et la quête de plus d'équité dans les filières. En cherchant à révéler les différentes représentations mentales (Gallen, 2005) que les individus se font du commerce équitable et de ses producteurs, notre étude exploratoire aura pour objectif d'éclairer l'hétérogénéité des motivations qui sous-tendent l'achat de produits issus du commerce équitable.
Méthodologie : une enquête exploratoire qualitative, puis quantitative

L'étude comporte deux phases de recueil de données: qualitative puis quantitative. Ayant pour objectif de révéler la diversité des représentations des consommateurs du commerce équitable et de ses producteurs, la première phase a été qualitative et exploratoire. Au total, 45 individus ont été enquêtés, selon deux méthodes qualitatives complémentaires. Vingt individus ont été interviewés en face à face à l'aide d'un guide d'entretien et 25 individus interviewés lors de trois focus groupes, organisés entre Montpellier et Paris. Nous avons prêté attention à l'hétérogénéité de l'échantillon et aux variables déterminantes qui se sont dégagées dans la revue de littérature : catégorie socioprofessionnelle, âge, sexe (Croutte et al., 2006), influence de l'expérience de comportements éthiques ou non (Berger et Kanetkar, 1995), l'engagement ou non dans des associations environnementales ou politiques... Dans cette première phase qualitative, les individus se sont exprimés en quatre temps sur leurs représentations, dans l'ordre : a) du concept de commerce équitable; b) des labels "Commerce équitable " (CE) ; c) des consommateurs des produits issus du CE, d) des producteurs du CE. Toutes les interviews et tous les focus groupes ont été retranscrits puis analysés de façon individuelle et thématique.

À l'issue de cette enquête qualitative, une enquête quantitative par questionnaire a été effectuée sur un échantillon de 228 individus. Le questionnaire abordait les points suivants :

- les représentations des consommateurs sur la qualité gustative des produits du CE, leur impact sur l'environnement, leur authenticité et enfin sur les représentations qu'ils ont des producteurs ;

- les motivations d'achat ou de non-achat de ces produits, par des questions projectives (Steinman, 2009);

- la définition et la signification de l'engagement pour les consommateurs ;

- l'expression de la sensibilité des individus à la mention "petits producteurs " et la justification qu'ils apportent à leurs réponses ; 
- la caractérisation des profils sociodémographiques des individus.

Les questionnaires ont été administrés par téléphone (146) ou en face à face (198), dans la région française du LanguedocRoussillon.

\section{Résultats}

Les analyses proviennent d'un aller-retour entre les résultats des deux phases, qualitative puis quantitative. Les premiers résultats nous permettent d'identifier ce qu'est le commerce équitable pour les individus, les différents vocabulaires qui le caractérisent et ses différentes représentations. Les deuxièmes apportent des informations sur la façon dont les individus se représentent les producteurs du commerce équitable. Enfin, les troisièmes, uniquement issus de la phase qualitative, permettent d'établir le lien entre les représentations que les consommateurs ont du commerce équitable et celles qu'ils ont de ses producteurs.

\section{Les différentes catégories de représentation du commerce équitable pour les consommateurs}

La phase qualitative a permis de distinguer cinq représentations du commerce équitable qui se différencient par leurs orientations plutôt altruistes ou plutôt individualistes: les deux premières sont des représentations d'ordre social ("réduction des inégalités sociales mondiales et soutien aux plus faibles") ou politique ("une alternative crédible au commerce conventionnel ") ; les trois der- nières sont d'ordre culturel ("un produit exotique produit selon un savoir-faire traditionnel"), naturel ("un produit meilleur pour ma santé et pour l'environnement ") ou liées au couple plaisir-tradition ("un produit de meilleur goût, authentique"). Pour les individus, ces représentations ne sont pas exclusives les unes des autres, ils les combinent souvent et les hiérarchisent selon leurs priorités et leurs valeurs sousjacentes. Il est intéressant de noter que les trois dernières représentations du commerce équitable ne reposent sur aucun de ses principes fondamentaux. Ainsi les représentations de produits naturels, authentiques, de meilleur goût, respectant l'environnement, voire même de produits biologiques, ne sont aucunement assurées par la présence d'un label CE mais seulement induites dans l'esprit du consommateur.

Dans l'enquête quantitative, sur 228 individus interrogés, 168 disent avoir déjà acheté des produits du commerce équitable, soit un taux de pénétration de $74 \%$ comparable au résultat de l'étude Ipsos de 2002 (77\%). Cela ne signifie par pour autant qu'il s'agit dans tous les cas d'acheteurs réguliers, mais montre que le terme "commerce équitable " est connu, plus que par ouï-dire, de plus des trois-quarts des personnes enquêtées.

L'existence de ces catégories de représentations a été testée au travers de questions sur les trois caractéristiques supposées citées dans l'étude qualitative par les consommateurs : le respect de l'environnement ; le couple " plaisir-tradition "; le caractère " naturel " des produits. Les résultats sont présentés dans le tableau 1.

Ces résultats montrent que les consommateurs voient, au-delà des bénéfices sociaux et économiques du commerce équitable, des avantages en termes d'environnement, de nature et d'authenticité. Parmi les individus enquêtés, $40 \%$ pensent que ces produits ont un meilleur goût. Si ces individus ne sont pas majoritaires, on constate tout de même que leur poids n'est pas négligeable et que le bon goût supposé des produits équitables pourrait peser dans le choix d'une partie des consommateurs.

À l'issue de l'analyse des entretiens qualitatifs, il apparaissait que les représentations diverses du CE étaient fortement liées à l'image des producteurs. L'enquête quantitative le confirme, puisque $78 \%$ des individus identifient les producteurs du CE à de "petits producteurs ", alors que seulement $11 \%$ des individus pensent qu'ils peuvent avoir n'importe quel profil.

\section{Deux représentations opposées de la production équitable}

Confirmant ce qu'a montré Ruwet (2007), notre analyse du discours des individus enquêtés en entretiens qualitatifs fait apparaitre deux représentations opposées de la production : la petite agriculture et l'agriculture industrielle. Ainsi, le vocabulaire se rapportant aux " petits producteurs " est très riche et fait appel à une dimension affective. Le consommateur idéalise la petite agriculture et cette idéalisation va de pair avec une "diabolisation" de l'industrie agroalimentaire, souvent appelée "la grosse machine". Lors des entretiens approfondis, l'agriculture industrielle a une image très négative auprès de la majorité des consommateurs. Elle est jugée responsable des récentes crises alimentaires, de la pollution et d'une uniformisation des produits alimentaires vers des produits néfastes pour la santé. Dans les représentations idéales, le CE reste associé à des modes

\section{Tableau 1. Les attributs associés aux produits du commerce équitable.}

Table 1. The attributes associated to fair trade products.

\begin{tabular}{|c|c|c|c|c|c|c|}
\hline \multirow{2}{*}{$\begin{array}{l}\text { Selon vous, les produits } \\
\text { issus du commerce } \\
\text { équitable: }\end{array}$} & \multicolumn{2}{|c|}{ Oui } & \multicolumn{2}{|c|}{ Non } & \multicolumn{2}{|c|}{ Ne se prononcent pas } \\
\hline & $\begin{array}{c}\% \text { des } \\
\text { individus } \\
\text { enquêtés }\end{array}$ & $\begin{array}{c}\text { Nombre } \\
\text { d'individus } \\
\text { (n=228) }\end{array}$ & $\begin{array}{l}\% \text { des } \\
\text { individus } \\
\text { enquêtés }\end{array}$ & $\begin{array}{c}\text { Nombre } \\
\text { d'individus } \\
\text { (n=228) }\end{array}$ & $\begin{array}{l}\% \text { des } \\
\text { individus } \\
\text { enquêtés }\end{array}$ & $\begin{array}{c}\text { Nombre } \\
\text { d'individus } \\
\text { (n= 228) }\end{array}$ \\
\hline $\begin{array}{l}\text { contribuent au respect } \\
\text { de l'environnement? }\end{array}$ & 67 & 153 & 16 & 37 & 17 & 38 \\
\hline ont un meilleur goût? & 40 & 91 & 42 & 95 & 18 & 42 \\
\hline $\begin{array}{l}\text { sont plus naturels, plus } \\
\text { authentiques? }\end{array}$ & 67 & 152 & 19 & 44 & 14 & 32 \\
\hline
\end{tabular}


Tableau 2. Les justifications de la sensibilité à la mention « Petits producteurs ".

Table 2. The reason of consumer sensitivity to the mention "Small Producers".

\begin{tabular}{|c|c|c|c|c|}
\hline $\begin{array}{l}\text { Selon vous, pourquoi êtes-vous } \\
\text { sensibles à la mention PP sur les } \\
\text { produits issus du CE ? }\end{array}$ & $\begin{array}{c}\text { "rassure sur les } \\
\text { bénéficiaires } \\
\text { du CE " }\end{array}$ & $\begin{array}{c}\text { "un produit plus artisanal, } \\
\text { plus authentique, } \\
\text { plus naturel " }\end{array}$ & $\begin{array}{c}\text { "le maintien } \\
\text { d'une tradition, } \\
\text { d'un savoir-faire " }\end{array}$ & Autre \\
\hline \% des individus sensibles à la mention PP & 50,9 & 23,4 & 17 & 8,7 \\
\hline $\begin{array}{l}\text { Nbre d'individus sensibles à la mention PP } \\
(n=228)\end{array}$ & 92 & 42 & 31 & 16 \\
\hline
\end{tabular}

CE : commerce équitable ; PP : petits producteurs.

traditionnels de production et à de petits agriculteurs que les personnes enquêtées imaginent dans des conditions précaires avec un métier difficile.

L'enquête par questionnaire montre que $78 \%$ des personnes interrogées qui achètent des produits du commerce équitable disent le faire pour s'engager et les deux tiers le justifient par leur volonté de soutenir ces petits producteurs. Deux niveaux de réflexion générale sur l'engagement vis-à-vis du commerce équitable ont été distingués: s'engager pour un commerce international plus équitable, de façon globale, ou s'engager pour soutenir les petits producteurs, à un niveau plus local. Le premier, plus abstrait, repose sur des principes idéologiques et politiques, ceux du changement d'un système de marché conventionnel jugé non satisfaisant. Le second, plus concret, repose sur des principes d'actions au niveau local, il est orienté vers des acteurs précis et identifiés : "les petits producteurs ". Ces niveaux de réflexion ne s'opposent pas, ils ne témoignent pas d'un engagement que l'on pourrait juger plus fort ou plus faible (ils peuvent être complémentaires), mais reposent sur des niveaux de réflexion générale différents. Au total, les consommateurs qui estiment s'engager par l'achat de produits CE le font à $32 \%$ pour un commerce international plus équitable et à $60 \%$ pour soutenir les "petits producteurs".

À la question "Etes-vous sensible ou pas du tout sensible à la mention "fabriqué par de petits producteurs?" sur les produits du CE?", $80 \%$ des individus se sont dit sensibles et $13 \%$ non sensibles. Il est intéressant de noter que, parmi les individus sensibles, $22 \%$ n'ont jamais acheté de produits issus du CE. La mention PP pourrait alors être un critère décisif dans une future décision d'achat de produits équitables pour ces consommateurs.
Lorsqu'on demande ensuite aux individus d'exprimer les raisons de leur sensibilité à cette mention PP, nous retrouvons les résultats illustrés dans le tableau 2.

La moitié des individus sensibles à la mention PP (51\%) se disent "rassurés sur les bénéficiaires $d u C E$ " grâce à cette mention; $23 \%$ identifient ces produits "comme plus artisanaux, plus authentiques, plus naturels " et enfin $17 \%$ affirment être sensibles à cette mention car elle témoigne du maintien d'une tradition et d'un savoir-faire local. Ces résultats recoupent ceux des entretiens qualitatifs, où les producteurs étaient vus comme les garants d'une culture rurale et d'une tradition forte, faisant preuve d'une attention particulière envers leurs produits, jugés de qualité supérieure. La mention PP est fortement valorisée par les consommateurs, car ils la jugent "plus claire", "plus transparente", "plus attirante". Certains consommateurs disent être plus convaincus par cette mention que par un label CE - comme Max Havelaar, souvent cité - qui inspire un certain scepticisme et est souvent assimilé à une marque.

\section{Quels liens \\ les consommateurs établissent-ils entre les mentions " commerce équitable " et " petits producteurs " ?}

Uniquement à partir de l'analyse des entretiens qualitatifs, nous avons croisé les représentations que les consommateurs ont des producteurs et de la mention "Commerce équitable ". Trois principaux profils de représentations peuvent être distingués (tableau 3).

Le premier profil, le plus largement répandu parmi les individus enquêtés, assimile clairement le commerce équitable aux petits producteurs. Ces personnes n'imaginent les agriculteurs du CE que comme de petits producteurs et font référence à deux motivations complémentaires : la première, altruiste, est le "soutien aux populations les plus défavorisées " (représentation d'ordre social), la seconde, plus égoïste, est "l'achat d'un produit de meilleure qualité " (représentation tradition-plaisir). Ces consommateurs valorisent davantage un produit qui est doublement signalé, car ces deux attributs sont perçus comme congruents. Ils se sentent rassurés sur les bénéficiaires de ce commerce et sur l'authenticité et le savoir-faire mis en œuvre dans la production. On comprend alors que ces consommateurs percevraient l'industrialisation des filières équitables comme une perte de sens et de qualité.

Le deuxième profil a une représentation plus nuancée des producteurs intégrés dans des filières équitables : ils sont idéalement paysans mais peuvent aussi être industriels. Ces consommateurs perçoivent une incohérence entre leurs attentes politiques en ce qui concerne le CE et la réalité ambiguë de la production qu'ils perçoivent. L'image des "petits producteurs " véhiculée par des photos, des dessins ou des mentions sur les paquets n'est perçue que comme une opération de marketing ne reposant sur aucune réalité. Il existe alors, pour l'individu, une incohérence entre les représentations "marketing" de la mention PP et la représentation idéale qu'il se fait de la production. Ce sont des consommateurs qui n'achètent pas ou peu de produits labellisés dans des grandes surfaces, mais qui s'engagent dans des modes de consommation plus militants.

Enfin, le troisième profil ne semble pas accorder d'importance à l'identité des producteurs. Ces consommateurs les imaginent comme très mécanisés, sur de grandes surfaces, pouvant avoir des salariés en grand nombre et un rendement important. Pour ces consommateurs, l'image de l'industrialisation n'est pas 
Tableau 3. Représentations que les consommateurs se font du mouvement " commerce équitable " et de ses producteurs.

Table 3. Consumer representations of fair trade and fair trade producers.

\begin{tabular}{llll}
\hline $\begin{array}{l}\text { Profils de } \\
\text { représentations }\end{array}$ & \multicolumn{2}{l}{$\begin{array}{l}\text { Représentations } \\
\text { des producteurs }\end{array}$} & $\begin{array}{l}\text { Représentations du } \\
\text { commerce équitable }\end{array}$ \\
\cline { 2 - 3 } & PP & IND & \\
\hline Premier profil & $X$ & & $\begin{array}{l}\text { Social et tradition-plaisir } \\
\text { Politique }\end{array}$ \\
Trouxième profil & $X$ & $X$ & Social \\
\hline
\end{tabular}

PP : petits producteurs; IND : production industrielle.

aussi négative que pour les précédents. Elle peut être un gage de sécurité sanitaire ou un moyen de développement efficace et inévitable des filières pour l'économie des pays du Sud. Ils recherchent avant tout, pendant leurs achats, un prix peu élevé et l'assurance d'une sécurité sanitaire minimale. Ils ne prêtent pas attention à la mention " $\mathrm{PP}$ "sur les produits. Ce sont typiquement des non-acheteurs de produits équitables ou des acheteurs occasionnels.

\section{Discussion \\ et conclusion}

Cette étude n'est pas exempte de limites et l'échantillon limité de la phase quantitative ne permet pas de généraliser les résultats. Cependant, que ce soit dans les entretiens approfondis ou dans l'enquête par questionnaire, on constate que la mention "Commerce équitable " n'est désormais plus uniquement reconnue par les consommateurs comme le signe de plus d'équité dans le commerce. Cette mention signale aussi une origine de petits producteurs, signes eux-mêmes d'un soin apporté à la production, d'une authenticité du produit et donc de sa qualité. La motivation des consommateurs pour les produits du commerce équitable n'est donc pas uniquement altruiste, mais aussi plus égoïste, ce d'autant plus qu'un désintérêt pour les questions d'équité du commerce international est sans doute difficile à avouer lors des enquêtes. Le relatif succès des produits équitables n'a donc pas pour seule origine la montée d'une revendication d'équité, mais est aussi lié à la recherche, par les consommateurs, de produits rassurants dans le cadre d'une suspicion vis-à-vis du secteur agroalimentaire standard. Si la notoriété du commerce équitable dépasse désormais $80 \%$ de la population (Alpha et al., 2008) et que les acheteurs réguliers sont en progression sur le marché, il serait donc abusif de considérer qu'il s'agit là seulement d'engagements éthiques. Il apparaît, en tout cas, un certain décalage entre des promoteurs du commerce équitable qui mettent en avant une revendication d'équité dans le commerce et des consommateurs qui peuvent aussi voir dans l'origine des produits des garanties de qualité sanitaire ou gustative.

Un tel résultat confirme qu'une labellisation équitable de grandes plantations, qui garantiraient une meilleure rémunération de leur main-d'œuvre, risque de faire perdre aux produits qui en seraient issus, l'avantage supplémentaire que leur confère la mention d'une origine de "petits producteurs". En terme commercial, on comprend donc la prudence des opérateurs du commerce équitable qui s'interrogent sur un tel élargissement de leurs fournisseurs. Le mouvement du CE est né et s'est développé en ciblant les producteurs paysans organisés en coopératives et bénéficie de l'image positive et idéalisée de ce type de producteurs. Labelliser d'autres types d'entreprises, plus industrielles, constituerait une sorte d'épreuve de vérité où seule resterait la garantie de rémunération des entreprises de production. Une telle option peut conduire à s'interroger sur la rémunération, non seulement de ces entreprises, mais aussi de celles qui interviennent plus en aval, dans la transformation du produit et dans sa distribution. Plus radicalement, cette option pourrait conduire à véritablement s'interroger sur la répartition de la valeur ajoutée entre les acteurs de la filière, en prenant ainsi plus à la lettre l'expression " commerce équitable".

Ce mouvement alternatif se retrouve aujourd'hui dans une position difficile, "contre et dans le marché" (Le Velly, 2006). Sa montée en puissance économique lui donne potentiellement un poids politique croissant pour espérer peser sur les règles du commerce international. Si son extension lui fait déjà prêter le flanc à la critique d'une récupération par la distribution standard et les grandes entreprises agroalimentaires, franchir le pas et associer d'avantage le commerce équitable à des entreprises plus industrielles fait courir le risque d'une critique plus affirmée et plus en amont de la filière, celle de pactiser avec les grandes entreprises de production.

\section{Références}

Alpha A, François M, Lagandré D. Le commerce équitable en France en 2007. Paris: ministère des Affaires étrangères et européennes ; Plate-forme pour le commerce équitable, 2008.

Berger IE, Kanetkar V. Increasing Environmental Sensitivity via Workplace Experiences. Journal of Policy and Marketing 1995; 14 : 205-15.

Boltanski L, Chiapello E. Le nouvel esprit du capitalisme. Paris: Gallimard, 1999.

Camus S. La marque authentique: approche cognitive expérientielle à partir de la littérature, des pratiques commerciales et des discours des consommateurs. Sixièmes journées normandes de la recherche sur la consommation: société et consommation. France : Groupe ESC Rouen, 2007.

Carù $A$, Cova $B$. Expériences de marque : comment favoriser l'immersion du consommateur ? Décisions Marketing 2006 ; 41 : 43-52.

Croutte P, Delpal F, Hatchuel G. Représentations et pratiques de la consommation engagée: évolution 2002. Cahier de Recherche du CREDOC 2006; 231: 1-145.

De Ferran F, Grunert KG. French fair trade coffee buyers' purchasing motives: An exploratory study using means-end chains analysis. Food Quality and Preference 2007; 18: 218-29.

Gallen C. Le rôle des représentations mentales dans le processus de choix, une approche pluridisciplinaire appliquée au cas des produits alimentaires. Recherche et Application en Marketing $2005 ; 20$ : 59-76.

Henson S, Traill B. Measuring perceived Performance of the Food System and Consumer Food-Related Welfare. Journal of Agricultural Economics 2000 ; 51 : 388-404.

Ipsos. La banque des sondages, 2002. http:// www.ipsos.fr/canalipsos/poll/7672.asp. 
Le Velly R. Le commerce équitable : des échanges marchands dans et contre le marché. Revue Française de Sociologie 2006; 47 : 319-40.

Micheletti M, Follesdal A, Stolle D. Politics, Products and Markets: Exploring Political Consumerism Past and Present. Londres: Transaction Publishers, 2004.

Ruwet C. What justification for sustainable consumption? In: Zaccaï E, ed. Sustainable consumption, ecology and fair trade. Londres : Routledge, 2007.
Seyfang G. Ecological citizenship and sustainable food consumption: Examining local organic food networks. Journal of Rural Studies $2006 ; 22$ : 383-95.

Steinman RB. Projective Techniques in Consumer Research. International Bulletin of Business Administration 2009 ; 5 : 37-45.

Stolle D, Hooghe M, Micheletti M. Politics in the supermarket: Political consumerism as a form of political participation. International political science review 2005 ; 26 : 245-69.
Tagbata D, Sirieix L. L'équitable, le bio et le goût. Quels sont les effets de la double labellisation bio-équitable sur le consentement à payer de consommateurs ? Cah Agri 2010 : epub ; doi : 10.1684/agr.2009.0371.

Warnier JP. Le paradoxe de la marchandise authentique. Imaginaire et consommation de masse. Paris : L'Harmattan, 1994. 